\title{
Anterocollis Worsened by Levodopa Therapy: A Case Report
}

\author{
Francesco Raudino, ${ }^{1, *}$ and Elisabetta Corengia ${ }^{1}$ \\ ${ }^{1}$ Department of Neurology Valduce Hospital, Via Dante n.11, Como, Italy \\ "Corresponding author: Francesco Raudino, Department of Neurology Valduce Hospital, Via Dante n.11, Como, Italy. E-mail: fraudino@hotmail.com
}

Received 2016 December 10; Accepted 2017 June 28.

\begin{abstract}
Introduction: Anterocollis was described in the Parkinson's disease and was often indicated as responding well to levodopa therapy. This article, on the other side, investigates the case of a patient with anterocollis whose condition worsened because of the levodopa therapy.

Case Presentation: A man suffering from Parkinson's disease since the age of 63 years began presenting a subacute appearance of anterocollis at the age of 73 years. Clonazepam, botulinum toxin, and physiotherapy relieved the pain without improving the anterocollis, while the suspension of both pramipexole and domperidone proved ineffective. The washout of the levodopa for 12 hours caused a worsening of the PD but an improvement of anterocollis. All attempts to increase the posology of the levodopa led to an improvement of the PD but worsening of the anterocollis.

Conclusions: Usually the anterocollis improves with levodopa, but in rare patients, levodopa therapy causes worsening of the anterocollis despite the improvement of rigidity.
\end{abstract}

Keywords: Anterocollis, Levodopa, Parkinson's Disease, Side Effect

\section{Introduction}

Quinn drew attention to the anterocollis in extrapyramidal diseases and defined it as "disproportionate anterocollis". At the beginning, he considered "disproportionate anterocollis" to be a peculiar sign of the multiple system atrophy after this had already been observed in association with some diseases and as a complication arising from specific drugs. In particular, "disproportionate anterocollis" was described in the Parkinson's disease (PD), and was often indicated as responding well to levodopa therapy. This article, on the other side, investigates the case of a patient with anterocollis whose condition worsened because of the levodopa therapy.

\section{Case Presentation}

A 63-year-old man with an unremarkable familial and personal anamnesis complained of micrography and motor impairment in the right limbs; PD was subsequently diagnosed and the patient begun levodopa therapy, showing a good response for several years. At the age of 69, pramipexole was added to the therapy to face a worsening motor impairment. At the age of 70, levodopa was replaced with levodopa/carbidopa/entacapone because of levodopa's wearing-off effects and because of dystonia in the right foot. At the age of 71 and over a period of two weeks, the patient developed a subacute appearance of anterocollis which was measured as grade 4 on the MDSUPDRS scale. The therapy was: levodopa $900 \mathrm{mg}$ a day; entacapone $800 \mathrm{mg}$ a day; pramipexole $2.10 \mathrm{mg}$ a day; and domperidone $30 \mathrm{mg}$ a day. The PD was subsequently measured as grade 2.5 on the Hoehn-Yarh's scale and grade 37 on the UPDRS part III scale; the patient was able to ameliorate the anterocollis applying a finger to her chin (geste antagoniste). The NMR of the cervical spine was unremarkable and the electromyography showed dystonia in the right scalene muscle as well as in both the scalene posterior muscles. On the contrary, serum creatinine kinase(CK) levels and thyroid functions were normal. One mg a day of clonazepam, botulinum toxin, and physiotherapy allowed pain relief without, however, improving the anterocollis. Suspending both pramipexole and domperidone equally proved ineffective. At the age of 73 , the patient was reassessed in hospital: a 12-hour washout of levodopa caused a worsening of the PD by $40 \%$ on the UPDRS part III scale but an improvement of anterocollis from 4 to 3 on the MDSUPDRS scale. To face a worsening PD, levodopa's posology was increased several times; however, along with the improvements of the $\mathrm{PD}$, the new posology also caused a worsening of the anterocollis. During the last control at the age of 79, the PD was under relative control by a levodopa dose of $800 \mathrm{mg}$ a day and an entacapone dose of $800 \mathrm{mg}$ a day. The anterocollis was controlled via botulinum toxin and physiotherapy. 


\section{Discussion}

Anterocollis is quite frequent in the PD: Kashihara et al, in a series of 356 patients, found no patient at the HoehnYahr's stage $1 ; 1.4 \%$ of patients at stage $2 ; 4.7 \%$ of patients at stage 3 ; and $9.1 \%$ of patients at stage 4 (1). Similarly, Fujmoto found anterocollis in 5.3\% of 131 patients with PD (2). The pathogenesis of anterocollis is debated: while it is considered to be a myopathy by some Authors, it is described as a dystonia by others (3). In our patient, the symptoms may relate to dystonia because of the absence of myopathic signs at the electromyography and because of the presence of the "geste antagoniste". Anterocollis may be provoked by some drugs but it has mostly been ascribed to dopaminergic drugs (3). The domperidone may also cause dystonia but, to our knowledge, there is no reference in the literature about cases of anterocollis related to its use. In our patient, the withdrawal of both drugs proved ineffective on the anterocollis. Usually anterocollis improves with levodopa, but in one of Yoshiyama's (4) patients and in four of Kashihara's (5), levodopa therapy was ineffective.
As in our case, in another of Yoshiyma's (4) patients, levodopa therapy caused the worsening of the anterocollis despite the rigidity improvement. As other parkinsonian symptoms that can be positively or negatively affected by levodopa, this suggests the involvment of different neurotrasmitters.

\section{References}

1. Kashihara K, Imamura T. Clinical correlates of anterior and lateral flexion of the thoracolumbar spine and dropped head in patients with Parkinson's disease. Parkinsonism Relat Disord. 2012;18(3):290-3.

2. Fujimoto K. Dropped head in Parkinson's disease. J Neurol. 2006;253 Suppl 7:VII21-6. doi: 10.1007/s00415-006-7006-3. [PubMed: 17131224].

3. van de Warrenburg BP, Cordivari C, Ryan AM, Phadke R, Holton JL, Bhatia KP, et al. The phenomenon of disproportionate antecollis in Parkinson's disease and multiple system atrophy. Mov Disord. 2007;22(16):2325-31. doi:10.1002/mds.21634. [PubMed: 17960814].

4. Yoshiyama Y, Takama J, Hattori T. The dropped head sign in parkinsonism. J Neurol Sci. 1999;167(1):22-5. [PubMed:10500257].

5. Kashihara K, Ohno M, Tomita S. Dropped head syndrome in Parkinson's disease. Mov Disord. 2006;21(8):1213-6. doi: 10.1002/mds.20948. [PubMed: 16703588]. 